\title{
Determination of Diethylstilbestrol in Water Samples by Dispersive Liquid-Liquid Microextraction Based on Solidification of Floating Organic Droplet Followed UV Spectrometry
}

\author{
Zongliang Niu ${ }^{1}$, Yingying Wen ${ }^{2 *}$ \\ ${ }^{1}$ Laboratory of Pathogenic Biology and Immunology, School of Basic Medical Science, Hainan Medical \\ University, Haikou 571199, China \\ ${ }^{2}$ Department of Environmental Science, School of Tropical and Laboratory Medicine, Hainan Medical \\ University, Haikou 571199, China
}

*Corresponding Author: Vingying Wen, Department of Environmental Science, School of Tropical and Laboratory Medicine, Hainan Medical University, Haikou 571199, China

\begin{abstract}
A dispersive liquid-liquid microextraction based on solidification of floating organic droplet (DLLME-SFO) combined with ultraviolet spectrometry (UV) method was developed for the determination of diethylstilbestrol (DES) in water samples. Several parameters including sample pH, extraction solution type and volume, dispersion solution type and volume, centrifugation speed and time were systematically investigated. The optimal extraction conditions of DLLME-SFO were: sample pH 3; extraction solution, 200 $\mu L 1$-dodecanol; dispersion solution, $400 \mu L A C N$; Centrifugation speed and time, 2500 rpmand 15 min. Under the optimum extraction and determination conditions, good responses for DES were obtained in a range of $0.5-$ $12.5 \mu \mathrm{g} / \mathrm{mL}$, with linear coefficients greater than 0.99. The recoveries of the DES ranged from $70 \%$ to $97 \%$. The limits of detection based on a signal-to-noise ratio of 3 was $0.15 \mu \mathrm{g} / \mathrm{mL}$. The method was applied to the determination of DES in tap and lake water samples with a satisfactory result. The results demonstrated that this DLLME-SFO-UV method was successfully applied to determine DES in water samples.
\end{abstract}

Keywords: Dispersive liquid-liquid microextraction based on solidification of floating organic droplet; Diethylstibestrol; Water samples; Ultraviolet

Abbreviations: ACN: acetonitrile; DES: diethylstilbestrol; DLLME-SFO: dispersive liquid-liquid microextraction based on solidification offloating organic droplet; MeOH: methanol; LOD: limit ofdetection; UV: ultraviolet.

\section{INTRODUCTION}

Diethylstilbestrol (DES), which is considered a representative example of an exogenous endocrine disrupting compound, is widely added in animal feed as growth promoter [1]. Nowadays, DES has been misused in order to promote the growth of animals. However, DES can be bio-accumulated in the food chain and remain in organisms for a long time, having a serious impact on the organism even at very low concentrations [1]. A number of reports have highlighted its potentially dangerous consequences to human and wild life, such as breast and prostate cancer [2,3]. In addition, it has been recognized that DES are widely present in the environmental waters, sediments and some aquatic matrices, through industrial, agricultural and municipal effluents [4]. Therefore, it is imperative to urgently develop simple, fast, and effective methods for monitoring and determining DES. In recent years, several extraction- determination methods have been developed for DES including solid phase extraction-high performance liquid chromatography [1], solid phase extraction-electrochemiluminescence [2], QuE ChERS-liquid chromatography-tandem mass spectrometry [3], dispersive liquid-liquid microextraction (DLLME)-capillary electrophoresis [4], gold nanoparticles immunoassay [5] and QuEChERS-ultraliquid chromatography-tandem mass spectrometry [6]. In these extraction methods, DLLME method is a novel miniaturized sample pre-treatment technique which requires smaller amounts of organic solvents and reduces the analysis cost more compared with other methods, such as LLE and SPE which have involved drawbacks (e.g. complicated, time-consuming procedures, large amounts of sample and organic solvents and difficulty). In DLLME, the appropriate mixture of extraction and disperser solvents 
is rapidly injected by syringe into an aqueous sample containing the analytes of interest. The fine particle of extracting solvent, which is dispersed into aqueous phase, allows its interaction with the analyte in automation. The advantages of the DLLME method are simplicity of operation, rapidity, low cost, high recovery and enrichment factors [7]. Recently, dispersive liquid-liquid microextraction based on solidification of floating organic droplet (DLLME-SFO) has been developed as a novel sample preparation technique, which follows the same principle as the DLLME technique [8,9]. In DLLMESFO procedures, a micro-drop of extraction solvent with low density and melting point near room temperature is delivered to the surface of an aqueous sample after centrifugation, and the extraction solvent is solidified at low temperature within the ice bath and collected easily [10].

In this study, we propose the use of DLLME-SFO for extraction of DES from two water samples, and ultraviolet spectrophotometry (UV) for determination of DES. To the best of our knowledge, this is the first demonstration for DLLME-SFO-UV for DES analysis.

\section{EXPERIMENTAL}

\subsection{Che micals and Materials}

Diethylstilbestrol (DES) was purchased from Sigma-Aldrich (Steinheim, Germany), 1-undecanol, 1dodecanol and 1-decanol were also from Sigma-Aldrich (Steinheim, Germany), High performance liquid chromatography-grade methanol $(\mathrm{MeOH})$ and acetonitrile $(\mathrm{ACN})$ were provided by $\mathrm{J} \& \mathrm{~K}$ Chemical (Beijing, China). $\mathrm{NaH}_{2} \mathrm{PO}_{4}, \mathrm{H}_{3} \mathrm{PO}_{4}, \mathrm{NaOH}$, and other affiliated chemicals were all obtained from Sinopharm Chemical Reagent Co. Ltd. (Shanghai, China). All solvents and chemicals were of analytical grade and used without further purification unless otherwise specified. HPLC-grade water was obtained by purifying demineralized water in a Milli-Q system (Millipore, Bedford, MA, USA), and was used throughout the work.

\subsection{Apparatus and Software}

Hitachi U-2910 UV-Vis spectrometry was provided by Hitachi Instrument Inc. (Hitachi, Japan). All the samples were passed through microporous nylon filters of $0.45 \mu \mathrm{m}$ pore sizes in diameter (Pall Corporation, USA). An Ion $510 \mathrm{pH}$ meter (Ayer Rajah Crescent, Singapore) was used to monitor $\mathrm{pH}$ adjustment. A centrifuge (Xiangyi, Hunan, China) was used for sample preparation.

\subsection{Pre paration of Standard and Sample}

Standard stock solution containing $1000 \mu \mathrm{g} / \mathrm{mL}$ of DES was prepared by dissolving the required amounts of the standard in $\mathrm{MeOH}$. It was stored in a refrigerator at $4{ }^{\circ} \mathrm{C}$. Working solutions were prepared from the stock solutions by dilution with appropriate amounts of Milli-Q water.

Lake water was collected from an artificial lake located in Longhua District of Haikou City (China). Tap water was obtained in the laboratory when needed. All the water samples were passed through microporous nylon filters with the pore sizes of $0.45 \mu \mathrm{m}$ in diameter. The samples were kept under refrigeration at $4{ }^{\circ} \mathrm{C}$ in the dark. Several aliquots from $8 \mathrm{~mL}$ filtered water samples were spiked with DES standard with different concentrations and followed by the DLLME-SFO procedure.

\subsection{DLLME-SFO Procedure}

Briefly, $8.00 \mathrm{~mL}$ of water sample was placed in a $10 \mathrm{~mL}$ of screw-cap glass tube with conic bottom and spiked with DES standard at $10 \mu \mathrm{g} / \mathrm{mL}$. Four hundred microliter $(400 \mu \mathrm{L})$ of ACN (as disperser solvent) containing $200 \mu \mathrm{L}$ 1-dodecanol (as extraction solvent) were rapidly injected into the sample solution with a $1.00 \mathrm{~mL}$ glass syringe and the mixture was gently shaken then ultrasonicated for $10 \mathrm{~min}$. In this step, a cloudy solution was formed and the DES in the water samples was extracted into fine droplets. Then, the mixture was centrifuged for $15 \mathrm{~min}$ at $2500 \mathrm{rpm}$. After centrifugation, the fine 1-dodecanol droplets floating on the top of the tube were cooled in an ice bath. The organic solvent was solidified and transferred into a conical vial by a small medicine spoon and then melted quickly at ambient conditions.

Finally, the extractant was redissolved in $1.5 \mathrm{~mL} \mathrm{MeOH}$ and analyzed by UV. The absorbance of the UV (A) was used as the index of extraction efficiency. 


\section{RES ULTS AND DIS CUSSION}

\subsection{DLLME-SFO Condition Optimization}

\subsubsection{Effect of Sample $\mathrm{pH}$}

It was well known that DES was diprotic acidic compound $\left(\mathrm{pK}_{\mathrm{a}, 1}=7.34\right.$ and $\mathrm{pK}_{\mathrm{a}, 2}=10.21$, respectively $)$ [11]. So, the $\mathrm{pH}$ of the sample has a great impact on the degree of ionization. As shown in Fig. 1, the absorbance of DES was the biggest when the sample $\mathrm{pH}$ was 3 . This was because the neutral species of DES were the dominant form when the sample $\mathrm{pH}$ was 3 , therefore DES was easily extracted to the 1dodecanol phase. Finally, pH 3 was selected as the optimum $\mathrm{pH}$ value of sample solution.

\subsubsection{Effect of Extraction Solution Type and Volume}

Different extraction solution will have different extraction efficiency. In this work, 1-undecanol and 1dodecanol were optimized. The result showed that the extraction efficiency of 1-dodecanol was higher than the extraction efficiency of 1-undecanol.

Increasing the volume of extraction solution increased the recovery of DES; however, the large volume of extraction solution decreased the concentration of DES and therefore resulted in low extraction efficiency. The result was shown in Fig. 2. When the extraction solution volume was $200 \mu \mathrm{L}$, the A of DES was the biggest. Therefore, two hundred microliter 1-dodecanol was selected as the extraction solution.

\subsubsection{Effect of Dispersion Solution Type and Volume}

Different dispersion solution will have different extraction efficiency. In this work, ACN and $\mathrm{MeOH}$ were optimized. The result showed that the extraction efficiency of ACN was higher than the extraction efficiency of $\mathrm{MeOH}$.

Increasing the volume of extraction solution increased the solubility of DES, this may help DES be extracted from sample to extraction solution; however, the large volume of dispersion solution decreased the concentration of DES and therefore resulted in low extraction efficiency. The result was shown in Fig. 3. When the extraction solution volume was $400 \mu \mathrm{L}$, the A of DES was the biggest. Therefore, four hundred microliter 1-dodecanol was selected as the extraction solution.

\subsubsection{Effect of Centrifugation Speed and Time}

Centrifugation could affect the separation of the extraction solution and the sample. In this work, 20004000 rpm and 5-20 min were optimized, the results were shown in Fig. 4 and 5. It can be seen that the extraction efficiency was best when the centrifugation speed and time were set at $2500 \mathrm{rpm}$ and $15 \mathrm{~min}$. So, we selected $2500 \mathrm{rpm}$ and $15 \mathrm{~min}$ as the optimized centrifugation speed and time.

Therefore, the optimized conditions for DLLME-SFO extraction of DES were attained as follows: sample $\mathrm{pH}$ 3; extraction solution, $200 \mu \mathrm{L}$ 1-dodecanol; dispersion solution, $400 \mu \mathrm{L}$ ACN; Centrifugation speed and time, $2500 \mathrm{rpm}$ and $15 \mathrm{~min}$.

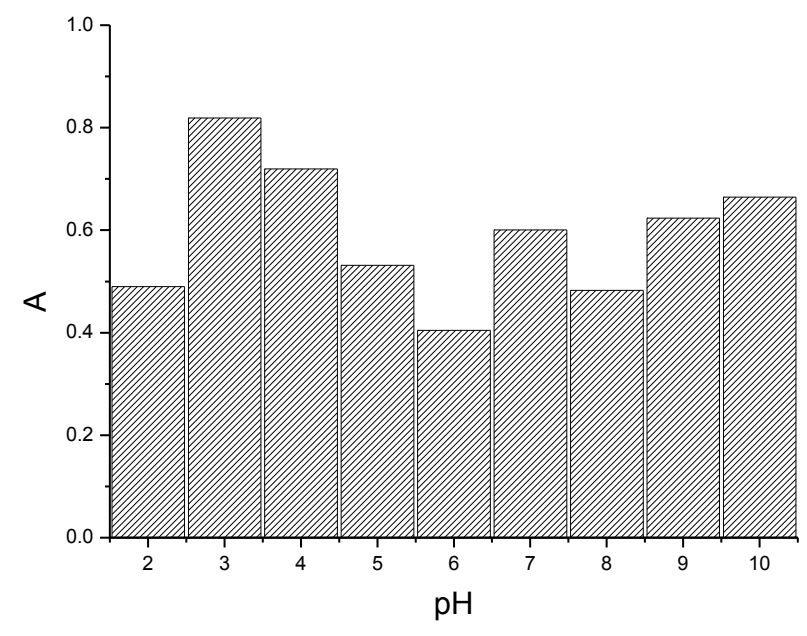

Fig1. Effect of sample $p H$ on extraction efficiency 


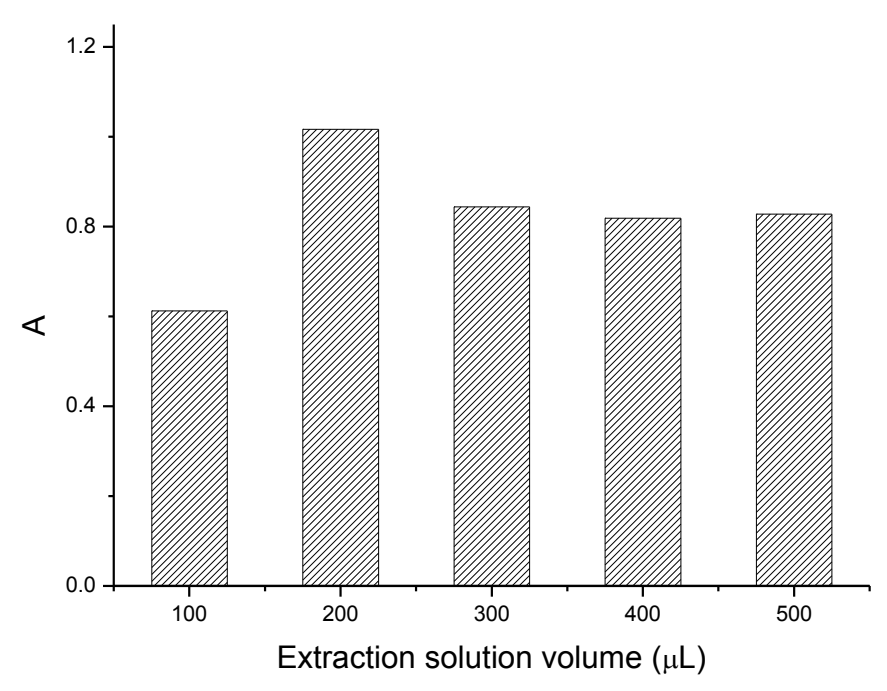

Fig2. Effect of extraction solution volume

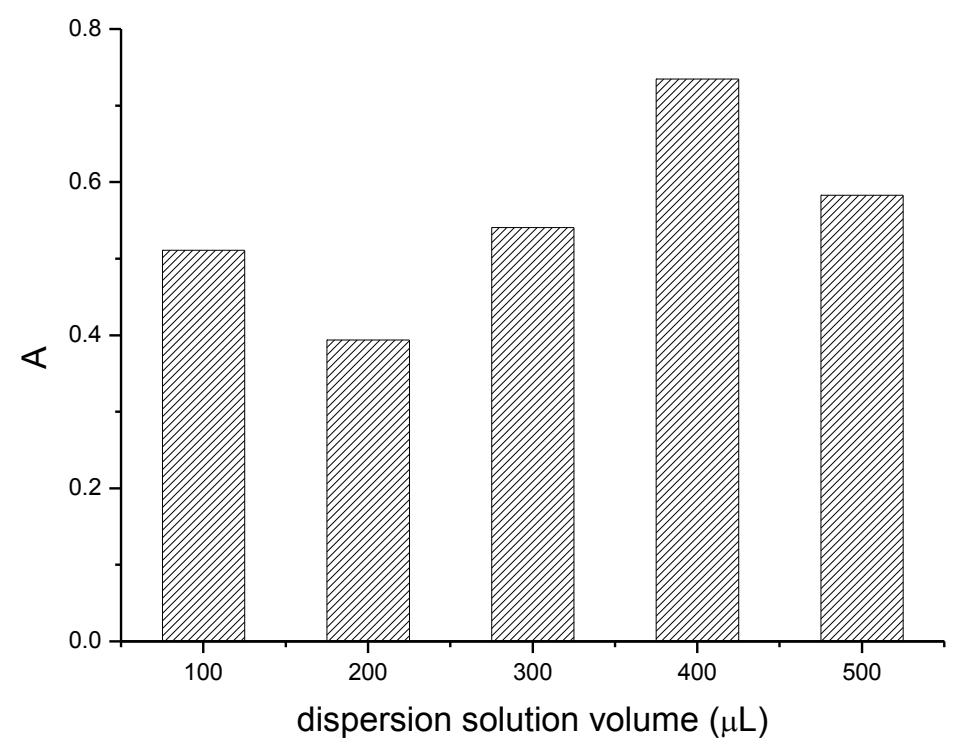

Fig3. Effect of dispersion solution volume

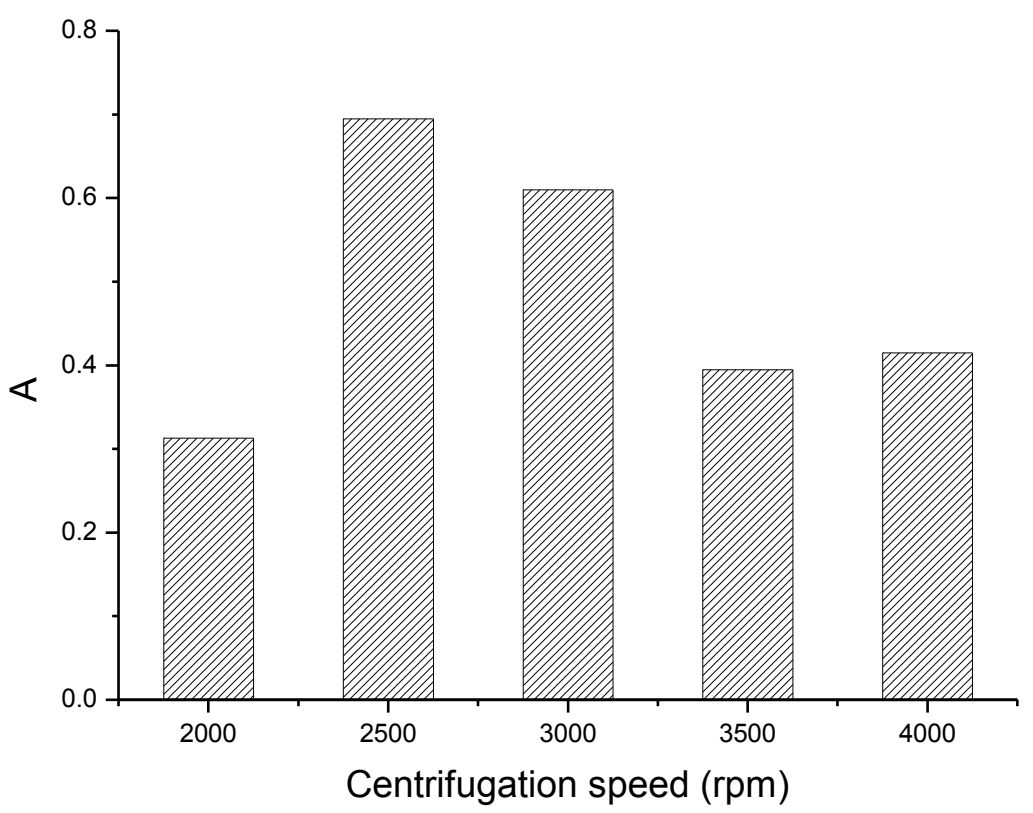

Fig4. Effect of centrifugation speed 


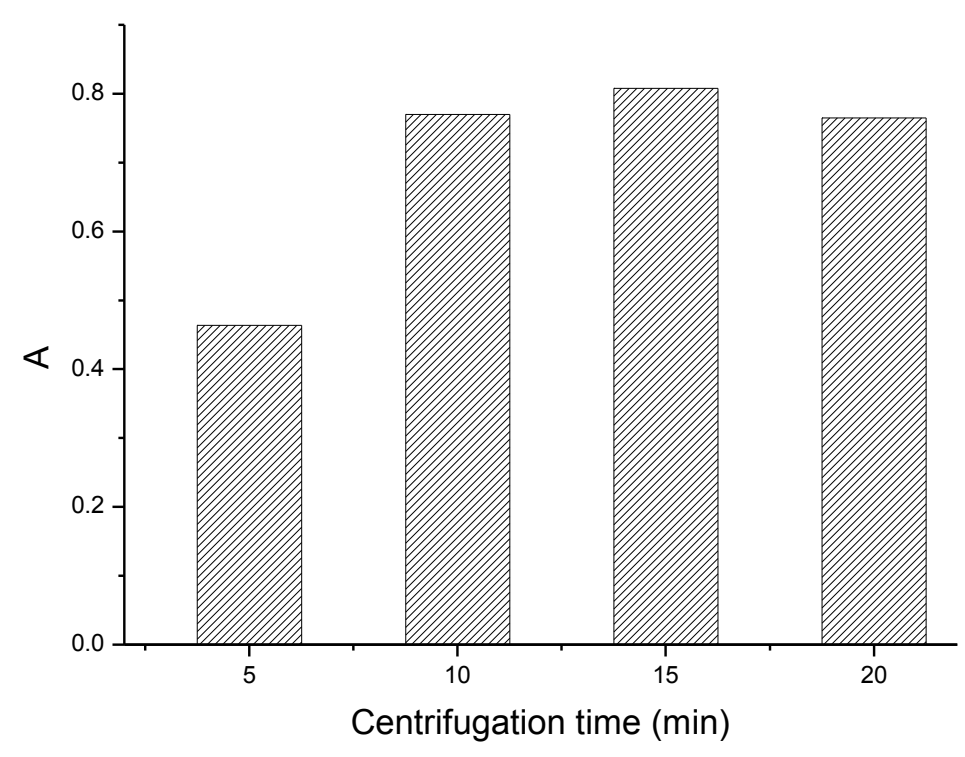

Fig5. Effect of centrifugation time

\subsection{Analytical Figures of Merit of the DLLME-SFO-UV Method}

The performance of the DLLME-SFO-UV method under optimal conditions was investigated. Linear correlation coefficients ( $\mathrm{r}$ ) assessed at six different concentrations were obtained between $\mathrm{A}$ and the corresponding concentrations of DES in the range of $0.5-12.5 \mu \mathrm{g} / \mathrm{mL}$, as shown in Table 1. Limit of detection (LOD) for the DES, calculated as the analyte concentration for which the value of A was three times the background noise $(3 \mathrm{~S} / \mathrm{N})$, was $0.15 \mu \mathrm{g} / \mathrm{mL}$ for the UV-Vis analysis.

\subsection{De te rmination of DES in Water Samples}

The developed DLLME-SFO-UV method was further applied to tap and lake water samples in order to check its practicality. The DES were not detected in the water samples. Recoveries were calculated for the spiked water samples with 1,2 and $5 \mu \mathrm{g} / \mathrm{mL}$ standards, respectively. The results are listed in Table 2. Satisfactory recoveries were obtained, ranged from $70 \%$ to $97 \%$. This validated the DLLME-SFOUV greatly applicable for the selective extraction, and accurate quantitation of trace DES in water samples.

Table1. Linear range, slope, intercept, correlation coefficients, and LOD for DES

\begin{tabular}{|c|c|c|c|c|}
\hline Analyte & Linear range $(\mu \mathrm{g} / \mathrm{mL})$ & Linear equation & Correlation coefficient $(\mathrm{r})$ & $\mathrm{LOD}(\mu \mathrm{g} / \mathrm{mL})$ \\
\hline DES & $0.5-12.5$ & $\mathrm{~A}=0.0506 \mathrm{C}+0.0306$ & 0.9910 & 0.15 \\
\hline
\end{tabular}

Table2. Recovery of DES from tap and lake water samples

\begin{tabular}{|c|c|c|c|}
\hline \multirow{2}{*}{ Analyte } & Added $(\mu \mathrm{g} / \mathrm{mL})$ & \multicolumn{2}{|c|}{ Recovery $(\%)$} \\
\cline { 3 - 4 } & & Lake water & Tap Water \\
\hline \multirow{2}{*}{ DES } & 1 & 79 & 86 \\
\cline { 2 - 4 } & 2 & 70 & 97 \\
\cline { 2 - 4 } & 5 & 70 & 76 \\
\hline
\end{tabular}

\section{CONClus ions}

In conclusion, a good, easy, and efficient method for the determination of DES in water samples was developed. The developed DLLME-SFO-UV offered wide linear range, good quantitative ability, and high precision. Also, this method used microliter extraction and dispersion solutions, was demonstrated to be a cost effective, and eco-friendly option for determination of DES in water samples.

\section{ACKNOWLEDGMENTS}

Financial support from the National Natural Science Foundation of China (81460328, 81660355), Research and Training Foundation of Hainan Medical University (HYCX2018003, HYCX2018016) and the Colleges and Universities Scientific Research Projects of the Education Department of Hainan Province (Hnky2018ZD-8) is gratefully acknowledged. 


\section{REFERENCES}

[1] Zhao W., Kang T., Lu L. and Cheng S., Magnetic surface molecularly imprinted poly(3-aminophenylboronic acid) for selective capture and determination of diethylstilbestrol, RSC Advances 8, 13129 (2018).

[2] Jiang Q., Zhang D., Cao Y. and Gan N., An antibody-free and signal-on type electrochemiluminescence sensor for diethylstilbestrol detection based on magnetic molecularly imprinted polymers-quantum dots labeled aptamer conjugated probes, J. Electroanal. Chem. 789, 1 (2017).

[3] Qin Y., Zhang J., Li Y., Han Y., Zou N., Jiang Y., Shan J. and Pan C., Multiplug filtration cleanup method with multi-walled carbon nanotubes for the analysis of malachite green, diethylstilbestrol residues, and their metabolites in aquatic products by liquid chromatography-tandem mass spectrometry, Anal. Bioanal. Chem. 408, 5801 (2016).

[4] Liu J., Lu W., Liu H., Wu X., Li J. and Chen L., Dispersive liquid-liquid microextraction for four phenolic environmental estrogens in water samples followed by determination using capillary electrophoresis, Electrophoresis 37, 2502 (2016).

[5] Liu X., Hu Y., Sheng X., Peng Y., Bai J., Lv Q., Jia H., Jiang H. and Gao Z., Rapid high-throughput detection of diethylstilbestrol by using the arrayed langasite crystal microbalance combined with gold nanoparticles through competitive immunoassay, Sensors and Actuators B 247, 245 (2017).

[6] Socas-Rodríguez B., Herrera-Herrera A. V., Hernández-Borges J. and Delgado M. Á. R., Multiresidue determination of estrogens in different dairy products by ultra-high performance liquid chromatography triple quadrupole mass spectrometry, J. Chromatogr. A 1496, 58 (2017).

[7] Wen Y., Li J., Zhang W. and Chen L., Dispersive liquid-liquid microextraction coupled with capillary electrophoresis for simultaneous determination of sulfonamides with the aid of experimental design, Electrophoresis 32, 2131 (2011).

[8] Shamsipur M., Fattahi N., Assadi Y., Sadeghi M. and Sharafi K., Speciation of As(III) and As(V)in water samples by graphite furnace atomic absorption spectrometry after solid phase extraction combined with dispersive liquid-liquid microextraction based on the solidification of floating organic drop, Talanta 130, 26 (2014).

[9] Zheng C., Zhao J., Bao P., Gao J. and He J., Dispersive liquid-liquid microextraction based on solidification of floating organic droplet followed by high-performance liquid chromatography with ultraviolet detection and liquid chromatography-tandem mass spectrometry for the determination of triclosan and 2,4dichlorophenol in water samples, J. Chromatogr. A 1218, 3830 (2011).

[10] Wang H., Yan H., Wang C., Chen F., Ma M., Wang W. and Wang X., Analysis of phenolic pollutants in human samples by high performance capillary electrophores is based on pretreatment of ultrasound-assisted emulsification microextraction and solidification of floating organic droplet, J. Chromatogr. A 1253,16 (2012).

[11] Wen Y., Li J., Liu J., Lu W., Ma J. and Chen L., Dual-cloud point extraction coupled to hydrodynamicelectrokinetic two-step injection followed by micellar electrokinetic chromatography for simultaneous determination of trace phenolic estrogens in water sample, Anal. Bioanal. Chem. 405, 5843 (2013).

Citation: Zongliang. Niu \& Yingying Wen, Determination of Diethylstilbestrol in Water Samples by Dispersive Liquid-Liquid Microextraction Based on Solidification of Floating Organic Droplet Followed UV Spectrometry," International Journal of Advanced Research in Chemical Science (IJARCS), vol. 5, no. 7, pp. 1-6, 2018. http://dx.doi.org/ 10.20431/2349-0403.0507001

Copyright: (C) 2018 Authors. This is an open-access article distributed under the terms of the Creative Commons Attribution License, which permits unrestricted use, distribution, and reproduction in any medium, provided the original author and source are credited. 